\title{
POSITIVE STABLE REALIZATIONS OF FRACTIONAL CONTINUOUS-TIME LINEAR SYSTEMS
}

\author{
TADEUSZ KACZOREK
}

Faculty of Electrical Engineering

Białystok Technical University, ul. Wiejska 45D, 15-351 Białystok, Poland

e-mail:kaczorek@isep.pw.edu.pl

\begin{abstract}
Conditions for the existence of positive stable realizations with system Metzler matrices for fractional continuous-time linear systems are established. A procedure based on the Gilbert method for computation of positive stable realizations of proper transfer matrices is proposed. It is shown that linear minimum-phase systems with real negative poles and zeros always have positive stable realizations.
\end{abstract}

Keywords: fractional, positive, stable, realization, system Metzler matrix, procedure, linear continuous-time.

\section{Introduction}

A dynamical system is called positive if its trajectory starting from any nonnegative initial state remains forever in the positive orthant for all nonnegative inputs. An overview of the state of the art in positive system theory is given in the monographs of Farina and Rinaldi (2000) as well as Kaczorek (2002). A variety of models having positive behavior can be found in engineering, economics, social sciences, biology and medicine, etc. An overview of the positive realization problem is given by Benvenuti and Farina (2004), Farina and Rinaldi (2000), or Kaczorek (2002; 2009b). The realization problem for positive continuous-time and discrete-time linear systems has been considered by Kaczorek (2004; 2006a; $2006 b$; 2006c) along with the positive minimal realization problem for singular discrete-time systems with delays (2005) as well as the realization problem for fractional linear systems $(2008 b ; 2011)$ and for positive 2D hybrid systems (2008c). Mathematical fundamentals of the fractional calculus and some applications are given by Kilbas et al. (2006) and Podlubny (1999), while some selected problems in fractional systems theory are presented by Kaczorek (2008a; 2009a; 2011). In this paper sufficient conditions will be established for the existence of positive stable realizations with the Metzler system matrix of fractional continuous-time linear systems and a procedure for computation of the realizations of proper transfer matrices will be proposed.

The paper is organized as follows. In Section 2 so- me definitions and theorems concerning fractional positive continuous-time linear systems are recalled and the problem formulation is given. A problem solution is presented in Sections 3 and 4. In Section 3, a procedure based on the Gilbert method is presented and in Section 4, the problem is solved for minimum-phase systems with negative real poles and zeros. Concluding remarks and open problems are presented in Section 5. The following notation is used: $\mathbb{R}$ means the set of real numbers, $\mathbb{R}^{n \times m}$ stands for the set of $n \times m$ real matrices, $\mathbb{R}_{+}^{n \times m}$ is the set of $n \times m$ matrices with nonnegative entries and $\mathbb{R}_{+}^{n}=\mathbb{R}_{+}^{n \times 1}$, $\mathbb{R}^{n \times m}[s]$ signifies the set of $n \times m$ polynomial matrices in $s$ with real coefficients, $M_{n}$ denotes the set of $n \times n$ Metzler matrices (real matrices with nonnegative off-diagonal entries), $I_{n}$ is the $n \times n$ identity matrix.

\section{Preliminaries and problem formulation}

Consider the continuous-time linear system

$$
\begin{aligned}
{ }_{0} D_{t}^{\alpha} x(t) & =A x(t)+B u(t), \quad 0<\alpha<1, \\
y(t) & =C x(t)+D u(t),
\end{aligned}
$$

where $x(t) \in \mathbb{R}^{n}, u(t) \in \mathbb{R}^{m}, y(t) \in \mathbb{R}^{p}$ are the state, input and output vectors, and $A \in \mathbb{R}^{n \times n}, B \in \mathbb{R}^{n \times m}$, $C \in \mathbb{R}^{p \times n}, D \in \mathbb{R}^{p \times m}$,

$$
\begin{aligned}
{ }_{0} D_{t}^{\alpha} x(t) & =\frac{\mathrm{d}^{\alpha} x(t)}{\mathrm{d} t^{\alpha}}=\frac{1}{\Gamma(1-\alpha)} \int_{0}^{t} \frac{\dot{x}(\tau)}{(t-\tau)^{\alpha}} \mathrm{d} \tau, \\
\dot{x}(\tau) & =\frac{\mathrm{d} x(\tau)}{\mathrm{d} \tau}
\end{aligned}
$$


is the Caputo definition of the $\alpha$-th order derivative, $\alpha \in$ $(0,1)$, while

$$
\Gamma(\alpha)=\int_{0}^{\infty} e^{-t} t^{\alpha-1} \mathrm{~d} t
$$

is the Euler gamma function.

Definition 1. (Kaczorek, 2011) The fractional system (1) is called (internally) positive if $x(t) \in \mathbb{R}_{+}^{n}, y(t) \in \mathbb{R}_{+}^{p}$, $t \geq 0$ for any initial conditions $x(0)=x_{0} \in \mathbb{R}_{+}^{n}$ and all inputs $u(t) \in \mathbb{R}_{+}^{m}, t \geq 0$.

Theorem 1. (Kaczorek, 2011) The fractional system (1) is positive if and only if

$$
A \in M_{n}, \quad B \in \mathbb{R}_{+}^{n \times m}, \quad C \in \mathbb{R}_{+}^{p \times n}, \quad D \in \mathbb{R}_{+}^{p \times m} .
$$

The transfer matrix of the fractional system (1) is given by

$$
\begin{aligned}
T\left(s^{\alpha}\right) & =C\left[I_{n} s^{\alpha}-A\right]^{-1} B+D \\
& =C\left[I_{n} \lambda-A\right]^{-1} B+D, \quad s^{\alpha}=\lambda .
\end{aligned}
$$

The transfer matrix is called proper if

$$
\lim _{\lambda \rightarrow \infty} T(\lambda)=K \in \mathbb{R}^{p \times m},
$$

and it is called strictly proper if $K=0$.

Definition 2. The matrices (4) are called a positive realization of the transfer matrix $T(\lambda) \in \mathbb{R}^{p \times m}(\lambda)$ if they satisfy Eqn. (5). The realization is called minimal if the dimension of $A$ is minimal among all realizations of $T(\lambda)$. The realization is called (asymptotically) stable if and only if all eigenvalues $\lambda_{i}$ of the matrix $A$ satisfy the conditions $\arg \lambda_{i}>\alpha \pi / 2, i=1, \ldots, n$.

The problem under consideration can be stated as follows: Given a rational matrix $T(\lambda) \in \mathbb{R}^{p \times m}(\lambda)$, find a positive stable realization with the system Metzler matrix $A$ of $T(\lambda)$, i.e.,

$$
A \in M_{n S}, \quad B \in \mathbb{R}_{+}^{n \times m}, \quad C \in \mathbb{R}_{+}^{p \times n}, \quad D \in \mathbb{R}_{+}^{p \times m},
$$

where $M_{n S}$ is the set of $n \times n$ (asymptotically) stable Metzler matrices.

\section{Problem solution}

Using the Gilbert method (Kaczorek, 1992), a procedure for finding positive stable realizations with system Metzler matrices will be presented for transfer matrices with real negative poles. Consider a stable positive continuoustime linear system (1) with a given proper transfer matrix of the form

$$
T(\lambda)=\left[\begin{array}{ccc}
T_{11}(\lambda) & \ldots & T_{1, m}(\lambda) \\
\vdots & \ldots & \vdots \\
T_{p, 1}(\lambda) & \ldots & T_{p, m}(\lambda)
\end{array}\right] \in \mathbb{R}^{p \times m}(\lambda)
$$

$$
T_{i, j}(\lambda)=\frac{n_{i, j}(\lambda)}{d_{i, j}(\lambda)}, \quad i=1, \ldots, p, \quad j=1, \ldots, m,
$$

where $\mathbb{R}^{p \times m}(\lambda)$ is the set of proper rational real matrices in $s^{\alpha}=\lambda$. The matrix $D$ can be found with the use of the formula

$$
D=\lim _{\lambda \rightarrow \infty} T(\lambda),
$$

and the strictly proper transfer matrix

$$
T_{s p}(\lambda)=T(\lambda)-D,
$$

which can be written in the form

$$
T_{s p}(\lambda)=\frac{N(\lambda)}{d(\lambda)} \in \mathbb{R}^{p \times m}(\lambda)
$$

where $N(\lambda) \in \mathbb{R}^{p \times m}[\lambda]$ and

$$
d(\lambda)=\lambda^{n}+a_{n-1} \lambda^{n-1}+\cdots+a_{1} \lambda+a_{0} .
$$

It is assumed that the equation $d(\lambda)=0$ has only distinct real negative roots $\lambda_{1}, \lambda_{2}, \ldots, \lambda_{n}\left(\lambda_{i} \neq \lambda_{j}\right.$ for $i \neq j)$, i.e., $d(\lambda)=\left(\lambda-\lambda_{1}\right)\left(\lambda-\lambda_{2}\right) \ldots\left(\lambda-\lambda_{n}\right)$. In this case, the transfer matrix (11) can be written in the form

$$
T_{s p}(\lambda)=\sum_{i=1}^{n} \frac{T_{i}}{\lambda-\lambda_{i}},
$$

where

$$
\begin{aligned}
& T_{i}=\lim _{\lambda \rightarrow \lambda_{i}}\left(\lambda-\lambda_{i}\right) T_{s p}(\lambda) \\
& =\frac{N\left(\lambda_{i}\right)}{\prod_{\substack{j=1 \\
j \neq i}}^{n}\left(\lambda_{i}-\lambda_{j}\right)}, \quad i=1, \ldots, n .
\end{aligned}
$$

Let

$$
\operatorname{rank} T_{i}=r_{i} \leq \min (p, m) .
$$

It is easy to show (Kaczorek, 1992) that

$$
T_{i}=C_{i} B_{i}, \quad \operatorname{rank} C_{i}=\operatorname{rank} B_{i}=r_{i},
$$
$i=1, \ldots, n$, where

$$
\begin{aligned}
C_{i} & =\left[\begin{array}{cccc}
C_{i, 1} & C_{i, 2} & \ldots & C_{i, r_{i}}
\end{array}\right] \in \mathbb{R}^{p \times r_{i}}, \\
B_{i} & =\left[\begin{array}{c}
B_{i, 1} \\
B_{i, 2} \\
\vdots \\
B_{i, r_{i}}
\end{array}\right] \in \mathbb{R}^{r_{i} \times m} .
\end{aligned}
$$

We shall show that the matrices are the desired positive stable realization with the system Metzler matrix

$$
\begin{aligned}
& A=\operatorname{blockdiag}\left[\begin{array}{lll}
I_{r_{1}} \lambda_{1} & \ldots & I_{r_{n}} \lambda_{n}
\end{array}\right], \\
& B=\left[\begin{array}{c}
B_{1} \\
\vdots \\
B_{n}
\end{array}\right], \quad C=\left[\begin{array}{lll}
C_{1} & \ldots & C_{n}
\end{array}\right] .
\end{aligned}
$$


Using (13), (16) and (17), we obtain

$$
\begin{aligned}
& T(\lambda) \\
& =C[I \lambda-A]^{-1} B \\
& =\left[\begin{array}{lll}
C_{1} & \ldots & C_{n}
\end{array}\right] \text { blockdiag }\left[I_{r_{1}}\left(\lambda-\lambda_{1}\right)^{-1}\right. \\
& \left.\ldots I_{r_{n}}\left(\lambda-\lambda_{n}\right)^{-1}\right]\left[\begin{array}{c}
B_{1} \\
\vdots \\
B_{n}
\end{array}\right] \\
& =\sum_{i=1}^{n} \frac{C_{i} B_{i}}{\lambda-\lambda_{i}}=\sum_{i=1}^{n} \frac{T_{i}}{\lambda-\lambda_{i}} .
\end{aligned}
$$

From (17), it follows that

(i) if $\lambda_{1}, \lambda_{2}, \ldots, \lambda_{n}$ are real negative, then the matrix $A$ is stable and is a Metzler matrix;

(ii) if

$$
T_{i} \in \mathbb{R}_{+}^{p \times m} \text { for } \quad i=1, \ldots, n,
$$

then we can choose

$$
C_{i} \in \mathbb{R}_{+}^{p \times r_{i}}, \quad B_{i} \in \mathbb{R}_{+}^{r_{i} \times m}
$$

for $i=1, \ldots, n$ and

$$
B \in \mathbb{R}_{+}^{\bar{n} \times m}, \quad C \in \mathbb{R}_{+}^{p \times \bar{n}}, \quad \bar{n}=\sum_{i=1}^{n} r_{i} .
$$

If $T(\infty) \in \mathbb{R}_{+}^{p \times m}$, then from (9) we have $D \in$ $\mathbb{R}_{+}^{p \times m}$. Therefore, the following theorem has been proved.

Theorem 2. There exists a positive stable realization (17), (9) of the proper transfer matrix (8) if the following conditions are satisfied:

(i) The poles of $T(\lambda)$ are distinct, real and negative, i.e., $\lambda_{i} \neq \lambda_{j}$ for $i \neq j, \lambda_{i}<0, i=1, \ldots, n$.

(ii) $T_{i} \in \mathbb{R}_{+}^{p \times m}$ for $i=1, \ldots, n$.

(iii) $T(\infty) \in \mathbb{R}_{+}^{p \times m}$.

If the conditions of Theorem 1 are satisfied, the following procedure can be used to find the desired positive stable realization with the system Metzler matrix.

\section{Procedure 1.}

Step 1. Using (9), find the matrix $D$ and the strictly proper transfer matrix (10) and write it in the form (11).

Step 2 . Find the real zeros $\lambda_{1}, \lambda_{2}, \ldots, \lambda_{n}$ of the polynomial (12).

Step 3. Using (14), find the matrices $T_{1}, \ldots, T_{n}$ and their decomposition (16).

Step 4. Using (17), find the matrices $A, B$ and $C$.
Example 1. Using Procedure 1, find a positive stable realization with the system Metzler matrix of the transfer matrix

$$
T(\lambda)=\left[\begin{array}{cc}
\frac{\lambda+3}{\lambda+1} & \frac{2 \lambda+5}{\lambda+2} \\
\frac{1}{\lambda+2} & \frac{\lambda+4}{\lambda+3}
\end{array}\right]
$$

Employing Procedure 1, we deliver the following. Using (9) for (20), we obtain

$$
D=\lim _{\lambda \rightarrow \infty} T(\lambda)=\left[\begin{array}{ll}
1 & 2 \\
0 & 1
\end{array}\right]
$$

and the strictly proper transfer matrix

$$
T_{s p}(\lambda)=T(\lambda)-D=\left[\begin{array}{cc}
\frac{2}{\lambda+1} & \frac{1}{\lambda+2} \\
\frac{1}{\lambda+2} & \frac{1}{\lambda+3}
\end{array}\right]
$$

which can be written in the form

$$
\begin{aligned}
& T_{s p}(s) \\
& =\frac{1}{(\lambda+1)(\lambda+2)(\lambda+3)} \\
& \quad \times\left[\begin{array}{rr}
2(\lambda+2)(\lambda+3) & (\lambda+1)(\lambda+3) \\
(\lambda+1)(\lambda+3) & (\lambda+2)(\lambda+1)
\end{array}\right] \\
& =\frac{N(\lambda)}{d(\lambda)} .
\end{aligned}
$$

In this case, $d(\lambda)=(\lambda+1)(\lambda+2)(\lambda+3), \lambda_{1}=-1$, $\lambda_{2}=-2, \lambda_{3}=-3$ and the condition (i) of Theorem 1 is met. Using (14) and (16), we obtain

$$
\begin{aligned}
T_{1}= & \frac{1}{(\lambda+2)(\lambda+3)} \\
& \times\left.\left[\begin{array}{rr}
2(\lambda+2)(\lambda+3) & (\lambda+1)(\lambda+3) \\
(\lambda+1)(\lambda+3) & (\lambda+2)(\lambda+1)
\end{array}\right]\right|_{\lambda=-1} \\
= & {\left[\begin{array}{ll}
2 & 0 \\
0 & 0
\end{array}\right], } \\
r_{1}= & \operatorname{rank} T_{1}=1, \quad T_{1}=C_{1} B_{1}, \\
B_{1}= & {\left[\begin{array}{ll}
1 & 0
\end{array}\right], \quad C_{1}=\left[\begin{array}{l}
2 \\
0
\end{array}\right], }
\end{aligned}
$$




$$
\begin{aligned}
T_{2}= & \frac{1}{(\lambda+1)(\lambda+3)} \\
& \times\left.\left[\begin{array}{cc}
2(\lambda+2)(\lambda+3) & (\lambda+1)(\lambda+3) \\
(\lambda+1)(\lambda+3) & (\lambda+2)(\lambda+1)
\end{array}\right]\right|_{\lambda=-2} \\
= & {\left[\begin{array}{ll}
0 & 1 \\
1 & 0
\end{array}\right], } \\
r_{2}= & \operatorname{rank} T_{2}=2, \quad T_{2}=C_{2} B_{2}, \\
B_{2}= & {\left[\begin{array}{ll}
B_{21} \\
B_{22}
\end{array}\right]=\left[\begin{array}{cc}
0 & 1 \\
1 & 0
\end{array}\right], } \\
C_{2}= & {\left[\begin{array}{ll}
C_{21} & C_{22}
\end{array}\right]=\left[\begin{array}{ll}
1 & 0 \\
0 & 1
\end{array}\right], } \\
T_{3}= & \frac{(24 \mathrm{~b})}{(\lambda+1)(\lambda+2)} \\
& \times\left.\left[\begin{array}{ll}
2(\lambda+2)(\lambda+3) & (\lambda+1)(\lambda+3) \\
(\lambda+1)(\lambda+3) & (\lambda+2)(\lambda+1)
\end{array}\right]\right|_{\lambda=-3} \\
= & {\left[\begin{array}{ll}
0 & 0 \\
0 & 1
\end{array}\right], } \\
r_{3}= & \operatorname{rank} T_{3}=1, \quad T_{3}=C_{3} B_{3}, \\
B_{3}= & {\left[\begin{array}{ll}
0 & 1
\end{array}\right], \quad C_{3}=\left[\begin{array}{l}
0 \\
1
\end{array}\right] . }
\end{aligned}
$$

From (23), it follows that the conditions (ii) of Theorem 1 are satisfied. Using (17) and (23), we obtain

$$
\begin{aligned}
A & =\left[\begin{array}{ccc}
I_{r_{1}} \lambda_{1} & 0 & 0 \\
0 & I_{r_{2}} \lambda_{2} & 0 \\
0 & 0 & I_{r_{1}} \lambda_{3}
\end{array}\right] \\
& =\left[\begin{array}{cccc}
-1 & 0 & 0 & 0 \\
0 & -2 & 0 & 0 \\
0 & 0 & -2 & 0 \\
0 & 0 & 0 & -3
\end{array}\right], \\
B & =\left[\begin{array}{c}
B_{1} \\
B_{2} \\
B_{3}
\end{array}\right]=\left[\begin{array}{cc}
1 & 0 \\
0 & 1 \\
1 & 0 \\
0 & 1
\end{array}\right], \\
C & =\left[\begin{array}{lllll}
C_{1} & C_{2} & C_{3}
\end{array}\right]=\left[\begin{array}{llll}
2 & 1 & 0 & 0 \\
0 & 0 & 1 & 1
\end{array}\right] .
\end{aligned}
$$

The desired positive stable realization of (20) is given by (25) and (22). This approach can be extended to transfer matrices with multiple real negative poles (Shaker and Dixon, 1977).

\section{Minimum-phase systems with real poles and zeros}

Consider the stable strictly proper irreducible transfer function

$$
T_{s p}(\lambda)=\frac{\bar{b}_{n-1} \lambda^{n-1}+\cdots+\bar{b}_{1} \lambda+\bar{b}_{0}}{\lambda^{n}+a_{n-1} \lambda^{n-1}+\cdots+a_{1} \lambda+a_{0}}
$$

$$
=\frac{\bar{b}_{n-1}\left(\lambda-z_{1}\right) \cdots\left(\lambda-z_{n-1}\right)}{\left(\lambda-\lambda_{1}\right)\left(\lambda-\lambda_{2}\right) \cdots\left(\lambda-\lambda_{n}\right)}
$$

where $\lambda_{1}, \ldots, \lambda_{n}$ are the real negative poles and $z_{1}, \ldots, z_{n-1}$ are real negative zeros of the transfer function.

Theorem 3. There exists a positive stable realization of (26) if

$$
\lambda_{k}<z_{k}<\lambda_{k+1} \quad \text { for } \quad k=1, \ldots, n-1 .
$$

Proof. From (14), we have

$$
\begin{aligned}
& T_{i} \\
& \quad=\frac{\left(\lambda_{i}-z_{1}\right)\left(\lambda_{i}-z_{2}\right) \ldots\left(\lambda_{i}-z_{n-1}\right)}{\left(\lambda_{i}-\lambda_{1}\right) \ldots\left(\lambda_{i}-\lambda_{i-1}\right)\left(\lambda_{i}-\lambda_{i+1}\right) \ldots\left(\lambda_{i}-\lambda_{n}\right)} \\
& \quad>0
\end{aligned}
$$

for $i=1, \ldots, n$ if the condition (ii) is satisfied. By Theorem 1 , the matrices

$$
\begin{aligned}
A & =\operatorname{diag}\left[\begin{array}{lll}
\lambda_{1} & \ldots & \lambda_{n}
\end{array}\right], \\
B & =\left[\begin{array}{c}
b_{1} \\
\vdots \\
b_{n}
\end{array}\right], \quad C=\left[\begin{array}{lll}
c_{1} & \ldots & c_{n}
\end{array}\right], \\
T_{i} & =b_{i} c_{i}, \quad i=1, \ldots, n
\end{aligned}
$$

are a positive stable realization of the transfer function 26.

Example 2. Let us find a positive realization of the strictly proper transfer function

$$
T_{s p}(\lambda)=\frac{\lambda+2}{\lambda^{2}+4 \lambda+3} .
$$

In this case, $\lambda_{1}=-1, \lambda_{2}=-3, z_{1}=-2$ and the condition (27) is satisfied. Using (14), we obtain

$$
T_{1}=\left.\frac{\lambda+2}{\lambda+3}\right|_{\lambda=-1}=\frac{1}{2}, \quad T_{2}=\left.\frac{\lambda+2}{\lambda+1}\right|_{\lambda=-3}=\frac{1}{2}
$$

and

$$
\begin{aligned}
& T_{1}=b_{1} c_{1}=\frac{1}{2}, \quad b_{1}=1, \quad c_{1}=\frac{1}{2}, \\
& T_{2}=b_{2} c_{2}=\frac{1}{2}, \quad b_{2}=1, \quad c_{2}=\frac{1}{2} \text {. }
\end{aligned}
$$

The desired positive realization has the form

$$
\begin{aligned}
& A=\left[\begin{array}{cc}
\lambda_{1} & 0 \\
0 & \lambda_{2}
\end{array}\right]=\left[\begin{array}{cc}
-1 & 0 \\
0 & -3
\end{array}\right], \\
& B=\left[\begin{array}{l}
b_{1} \\
b_{2}
\end{array}\right]=\left[\begin{array}{l}
1 \\
1
\end{array}\right] \\
& C=\left[\begin{array}{ll}
c_{1} & c_{2}
\end{array}\right]=\left[\begin{array}{ll}
\frac{1}{2} & \frac{1}{2}
\end{array}\right] .
\end{aligned}
$$


Now let us consider the strictly proper transfer matrix (11) rewritten in the form

$$
\begin{aligned}
& T_{s p}(\lambda)=\frac{1}{\left(\lambda-\lambda_{1}\right) \cdots\left(\lambda-\lambda_{n}\right)} \\
& \times\left[\begin{array}{cc}
\left(\lambda-z_{11}^{1}\right) \cdots\left(\lambda-z_{11}^{n_{11}}\right) & \cdots \\
\vdots & \cdots \\
\left(\lambda-z_{p, 1}^{1}\right) \cdots\left(\lambda-z_{p, 1}^{n_{p, 1}}\right) & \cdots
\end{array}\right. \\
& \left.\begin{array}{c}
\left(\lambda-z_{1, m}^{1}\right) \cdots\left(\lambda-z_{1, m}^{n_{1, m}}\right) \\
\vdots \\
\left(\lambda-z_{p, m}^{1}\right) \cdots\left(\lambda-z_{p, m}^{n_{p, m}}\right)
\end{array}\right] .
\end{aligned}
$$

where $\lambda_{1}, \ldots, \lambda_{n}$ are real negative poles and $z_{i j}^{k}, \quad i=$ $1, \ldots, p, \quad j=1, \ldots, m, \quad k=1, \ldots, n_{i j}$ are real negative zeros.

Theorem 4. There exists a positive stable realization (17) of 32 if

$$
\lambda_{k} \leq z_{i, j}^{k} \leq \lambda_{k+1}
$$

for $i=1, \ldots, p, j=1, \ldots, m$, and $k=1, \ldots, n_{i j}$.

The proof is similar to that of Theorem 3 . If the condition (33) is satisfied, then a positive stable realization (17) of (32) can be found with the use of Procedure 1.

Example 3. Using Procedure 1, we wish to find a positive realization of the strictly proper transfer matrix

$$
\begin{aligned}
T_{s p}(\lambda)= & \frac{1}{(\lambda+1)(\lambda+3)(\lambda+5)} \\
& \times\left[\begin{array}{ll}
(\lambda+2)(\lambda+4) & (\lambda+1)(\lambda+4) \\
(\lambda+2)(\lambda+5) & (\lambda+2)(\lambda+4)
\end{array}\right] .
\end{aligned}
$$

In this case, we have $\lambda_{1}=-1, \lambda_{2}=-3, \lambda_{3}=-5$, $z_{11}^{1}=-2, z_{11}^{2}=-4, z_{12}^{1}=-1, z_{12}^{2}=-4, z_{21}^{1}=-2$, $z_{21}^{2}=-5, z_{22}^{1}=-2, z_{22}^{2}=-4$, and the conditions (33) are satisfied. Therefore, by Theorem 4 , there exists a positive stable realization of the transfer matrix (34). Using (14) and (34), we obtain

$$
\begin{aligned}
T_{1}= & \frac{1}{(\lambda+3)(\lambda+5)} \\
& \times\left.\left[\begin{array}{ll}
(\lambda+2)(\lambda+4) & (\lambda+1)(\lambda+4) \\
(\lambda+2)(\lambda+5) & (\lambda+2)(\lambda+4)
\end{array}\right]\right|_{\lambda=-1} \\
= & {\left[\begin{array}{ll}
\frac{3}{8} & 0 \\
\frac{1}{2} & \frac{3}{8}
\end{array}\right] }
\end{aligned}
$$

$\operatorname{rank} T_{1}=2, \quad T_{1}=C_{1} B_{1}$,

$$
C_{1}=\left[\begin{array}{cc}
\frac{3}{8} & 0 \\
\frac{1}{2} & \frac{3}{8}
\end{array}\right], \quad B_{1}=\left[\begin{array}{ll}
1 & 0 \\
0 & 1
\end{array}\right] \text {, }
$$

$$
\begin{aligned}
T_{2}= & \frac{1}{(\lambda+1)(\lambda+5)} \\
& \times\left.\left[\begin{array}{ll}
(\lambda+2)(\lambda+4) & (\lambda+1)(\lambda+4) \\
(\lambda+2)(\lambda+5) & (\lambda+2)(\lambda+4)
\end{array}\right]\right|_{\lambda=-3} \\
= & {\left[\begin{array}{ll}
\frac{1}{4} & \frac{1}{2} \\
\frac{1}{2} & \frac{1}{4}
\end{array}\right] }
\end{aligned}
$$$$
\operatorname{rank} T_{2}=2, \quad T_{2}=C_{2} B_{2},
$$$$
C_{2}=\left[\begin{array}{cc}
\frac{1}{4} & \frac{1}{2} \\
\frac{1}{2} & \frac{1}{4}
\end{array}\right], \quad B_{2}=\left[\begin{array}{ll}
1 & 0 \\
0 & 1
\end{array}\right] \text {, }
$$

$$
\begin{aligned}
T_{3}= & \frac{1}{(\lambda+1)(\lambda+3)} \\
& \times\left.\left[\begin{array}{rr}
(\lambda+2)(\lambda+4) & (\lambda+1)(\lambda+4) \\
(\lambda+2)(\lambda+5) & (\lambda+2)(\lambda+4)
\end{array}\right]\right|_{\lambda=-5} \\
& =\left[\begin{array}{ll}
\frac{3}{8} & \frac{1}{2} \\
0 & \frac{3}{8}
\end{array}\right], \\
& \operatorname{rank} T_{3}=2, \quad T_{3}=C_{3} B_{3}, \\
C_{3} & =\left[\begin{array}{cc}
\frac{3}{8} & \frac{1}{2} \\
0 & \frac{3}{8}
\end{array}\right], \quad B_{3}=\left[\begin{array}{ll}
1 & 0 \\
0 & 1
\end{array}\right]
\end{aligned}
$$

The desired positive stable realization has the form

$$
\begin{aligned}
A & =\left[\begin{array}{cccc}
I_{r_{1}} \lambda_{1} & 0 & 0 \\
0 & I_{r_{2}} \lambda_{2} & 0 \\
0 & 0 & I_{r_{3}} \lambda_{3}
\end{array}\right] \\
& =\left[\begin{array}{cccccc}
-1 & 0 & 0 & 0 & 0 & 0 \\
0 & -1 & 0 & 0 & 0 & 0 \\
0 & 0 & -3 & 0 & 0 & 0 \\
0 & 0 & 0 & -3 & 0 & 0 \\
0 & 0 & 0 & 0 & -5 & 0 \\
0 & 0 & 0 & 0 & 0 & -5
\end{array}\right], \\
B & {\left[\begin{array}{l}
B_{1} \\
B_{2} \\
B_{3}
\end{array}\right]=\left[\begin{array}{cc}
1 & 0 \\
0 & 1 \\
1 & 0 \\
0 & 1 \\
1 & 0 \\
0 & 1
\end{array}\right], }
\end{aligned}
$$




$$
C=\left[\begin{array}{lll}
C_{1} & C_{2} & C_{3}
\end{array}\right]=\frac{1}{8}\left[\begin{array}{cccccc}
3 & 0 & 2 & 4 & 3 & 4 \\
4 & 3 & 4 & 2 & 0 & 3
\end{array}\right]
$$

\section{Concluding remarks}

Conditions for the existence of positive stable realizations with system Metzler matrices of fractional continuoustime linear systems have been established (Theorem 2). A procedure based on the Gilbert method for computation of positive stable realizations of proper transfer matrices has been proposed. It has been shown that minimum-phase systems with real negative poles and zeros always have the positive stable realizations (Theorems 3 and 4). The deliberations have been illustrated by numerical examples.

The following are open problems for fractional continuous-time linear systems:

1. Find necessary and sufficient conditions for the existence of positive stable realizations with system Metzler matrices of proper transfer matrices.

2. Give a method for finding positive stable realizations with system Metzler matrices which is not based on the Gilbert method of proper transfer matrices.

\section{Acknowledgment}

This work was supported by the Ministry of Science and Higher Education in Poland under the grant no. G/WE/1/11.

\section{References}

Benvenuti, L. and Farina, L. (2004). A tutorial on the positive realization problem, IEEE Transactions on Control 49(5): 651-664.

Farina, L. and Rinaldi, S. (2000). Positive Linear Systems, Theory and Applications, J. Wiley, New York, NY.

Kaczorek, T. (1992). Linear Control Systems, Vol. 1, Research Studies Press, J. Wiley, New York, NY.

Kaczorek, T. (2002). Positive $1 D$ and 2D Systems, SpringerVerlag, London.

Kaczorek, T. (2004). Realization problem for positive discretetime systems with delay, System Science 30(4): 117-130.

Kaczorek, T. (2005). Positive minimal realizations for singular discrete-time systems with delays in state and delays in control, Bulletin of the Polish Academy of Sciences: Technical Siences 53(3): 293-298.

Kaczorek, T. (2006a). A realization problem for positive continuous-time systems with reduced numbers of delays, International Journal of Applied Mathematics and Computer Science 16 (3): 325-331.
Kaczorek, T. (2006b). Computation of realizations of discretetime cone systems, Bulletin of the Polish Academy of Sciences: Technical Siences 54(3): 347-350.

Kaczorek, T. (2006c). Realization problem for positive multivariable discrete-time linear systems with delays in the state vector and inputs, International Journal of Applied Mathematics and Computer Science 16(2): 169-174.

Kaczorek, T. (2008a). Fractional positive continuous-time linear systems and their reachability, International Journal of Applied Mathematics and Computer Science 18(2): 223 228, DOI: $10.2478 / \mathrm{v} 10006-008-0020-0$.

Kaczorek, T. (2008b). Realization problem for fractional continuous-time systems, Archives of Control Sciences 18(1): 43-58.

Kaczorek, T. (2008c). Realization problem for positive 2D hybrid systems, COMPEL 27(3): 613-623.

Kaczorek, T. (2009a). Fractional positive linear systems, Kybernetes: The International Journal of Systems \& Cybernetics 38 (7/8): 1059-1078.

Kaczorek, T. (2009b). Polynomial and Rational Matrices, Springer-Verlag, London.

Kaczorek, T. (2011). Selected Problems in Fractional Systems Theory, Springer-Verlag, Berlin.

Kilbas, A.A., Srivastava, H.M. and Trujillo, J.J. (2006). Theory and Applications of Fractional Differential Equations, North-Holland, Amsterdam.

Podlubny, I. (1999). Fractional Differential Equations, Academic Press, San Diego, CA.

Shaker, U. and Dixon, M. (1977). Generalized minimal realization of transfer-function matrices, International Journal of Control 25(5): 785-803.

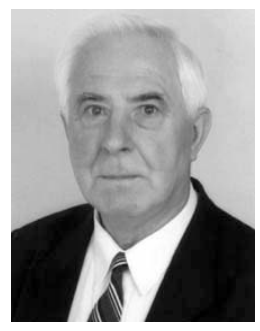

Tadeusz Kaczorek received the M.Sc., Ph.D and D.Sc. degrees in electrical engineering from the Warsaw University of Technology in 1956, 1962 and 1964, respectively. In the years 1968-69 he was the dean of the Electrical Engineering Faculty, and in the period of 1970-73 he was a deputy rector of the Warsaw University of Technology. In 1971 he became a professor and in 1974 a full professor at the same university. Since 2003 he has been a professor at Białystok Technical University. In 1986 he was elected a corresponding member and in 1996 a full member of the Polish Academy of Sciences. In the years 1988-1991 he was the director of the Research Centre of the Polish Academy of Sciences in Rome. In 2004 he was elected an honorary member of the Hungarian Academy of Sciences. He has been granted honorary doctorates by several universities. His research interests cover the theory of systems and automatic control systems theory, especially singular multidimensional systems, positive multidimensional systems, and singular positive $1 \mathrm{D}$ and $2 \mathrm{D}$ systems. He initiated research in the field of singular 2D and positive 2D systems. He has published 24 books (six in English) and over 950 scientific papers. He has also supervised 69 Ph.D. theses. He is the editor-in-chief of the Bulletin of the Polish Academy of Sciences: Technical Sciences and a member of editorial boards of ten international journals.

Received: 20 April 2011

Revised: 26 May 2011 